\section{Diferenciais entre homens e mulheres na mortalidade evitável no Brasil (1983-2005)}

\author{
Gender differences in avoidable mortality \\ in Brazil (1983-2005)
}

\begin{abstract}
${ }^{1}$ Faculdade de Medicina, Universidade Federal de Minas Gerais, Belo Horizonte, Brasil.

2 Instituto de Ciências Exatas, Universidade Federal de Minas Gerais, Belo Horizonte, Brasil.

Correspondência D. M. X. Abreu

Programa de Pós-graduação em Saúde Pública, Faculdade de Medicina, Universidade Federal de Minas Gerais. Av. Alfredo Balena 190, Belo Horizonte, $M G$ 30130-100, Brasil. dmxa@medicina.ufmg.br
\end{abstract}

\section{Abstract}

The aim of the article was to analyze gender differences in mortality in 117 Brazilian municipalities from 1983 to 2005, based on three groups of causes of avoidable death: (1) avoidable through early diagnosis and treatment, (2) avoidable by improvements in quality of treatment and medical care, and (3) ischemic heart disease. The association between avoidable mortality and demographic and socioeconomic conditions and healthcare variables was analyzed through negative binomial regression. The multiple decrement technique was used to evaluate the impact of avoidable causes on life expectancy for men and women. Men showed a higher risk of death for all three groups of avoidable causes, after controlling for selected variables. Women would gain more than men, with an increase of up to five years in life expectancy, if avoidable causes were eliminated by diagnosis and early treatment. Further research is needed in gender-related factors, which may be related to differential mortality rates in men and women.

Mortality; Causes of Death; Men; Women
Daisy Maria Xavier de Abreu 1

Cibele Comini César 2

Elisabeth Barboza França ${ }^{1}$

\section{Introdução}

Estudos sobre diferenciais de mortalidade entre homens e mulheres apontam para o fenômeno de que a população feminina experimenta taxas mais baixas de mortalidade geral e também por causas de morte específicas 1,2 . As razões para isso têm sido creditadas às diferenças biológicas e à adoção de estilos de vida distintos relacionados a questões de gênero (sexo construído culturalmente) 3,4 .

A sobremortalidade masculina é observada especialmente na população adulta jovem, tanto no mundo desenvolvido como em regiões em desenvolvimento 3,5. Tal fato tem sido avaliado freqüentemente por um indicador utilizado para medir os diferenciais entre sexos que é a esperança de vida ao nascer. No Brasil, em 2000, a esperança de vida ao nascer era de 66,7 anos para os homens e de 74,4 para as mulheres, com um diferencial a favor da população feminina de 7,6 anos. Apesar das tendências de redução dos níveis de mortalidade e aumento da esperança de vida ao nascer, observa-se que em 2005 a diferença entre os sexos não se alterou, já que a esperança de vida ao nascer para os homens atingiu 68,2 anos e para as mulheres 75,8 6 .

Análises sobre a estrutura de mortalidade por causas de morte podem auxiliar no entendimento do comportamento da mortalidade de homens e mulheres. A identificação dos grupos de causas que contribuíram para o declínio da 
mortalidade possibilita conhecer melhor quais as que podem influenciar na redução ou ampliação dos diferenciais entre os sexos 7. A utilização de listas de classificação de causas de morte serve como ferramenta para a avaliação dos riscos diferenciados para cada sexo. Nesse aspecto, vale considerar abordagens como a da mortalidade evitável, que se propõe a analisar as mortes que não deveriam ocorrer na presença de uma eficaz e preventiva ação de saúde ${ }^{8}$.

A mortalidade por causas evitáveis representa um indicador de deficiências potenciais na atenção à saúde, e sua análise pode indicar se há diferenças que sugerem benefícios maiores para um dos sexos, frente ao avanço das medidas de saúde pública e do conhecimento médico ${ }^{9}$. Mas, falta investigar se, ao controlar fatores como condições sócio-econômicas, idade, oferta e acesso aos serviços de saúde, o risco de morrer para a população é diferenciado segundo sexo, e para quais causas de morte observa-se um diferencial mais expressivo.

No Brasil, uma análise da mortalidade por causas evitáveis por atenção à saúde deve considerar o fato de que nas últimas décadas o contexto brasileiro sofreu mudanças significativas, que possivelmente repercutiram sobre os indicadores de saúde. Particularmente, ocorreram importantes transformações no modelo assistencial de saúde com a implantação do Sistema Único de Saúde (SUS), orientado por diretrizes de descentralização, regionalização e hierarquização dos serviços, com prioridade para ações de promoção e prevenção de saúde e ênfase no desenvolvimento de mecanismos de participação da população 10. Estudos sugerem que é possível creditar em parte ao modelo implementado pelo SUS a redução dos níveis de mortalidade evitável verificada no período 1983-2002 11 .

Face a essas questões, o presente estudo tem como objetivo analisar os diferenciais de mortalidade nas populações masculina e feminina brasileiras no período de 1983 a 2005, com ênfase nas causas de morte evitáveis por atenção à saúde. O período analisado abarca uma fase decisiva do processo de mudanças na organização do sistema de saúde no país, fator relevante para a análise das causas evitáveis por atenção à saúde que representa um indicador indireto da qualidade da assistência prestada.

\section{Material e métodos}

Tendo em vista o interesse em analisar o impacto de variáveis de contexto, a unidade de análise utilizada foi o município. A principal fonte de dados foi o Sistema de Informações sobre Mortalidade
(SIM), do Departamento de Informática do SUS (DATASUS) do Ministério da Saúde, para obtenção das informações sobre óbitos no período de 1983 a 2005. Para as populações masculina e feminina foram utilizadas as estimativas elaboradas pelo Instituto Brasileiro de Geografia e Estatística (IBGE), disponibilizadas pelo DATASUS por idade. A fonte de dados usada para pesquisar as variáveis sócio-econômicas dos municípios foi o Atlas de Desenvolvimento Humano, para 1991 e 2000, elaborado pelo Programa das Nações Unidas para o Desenvolvimento (PNUD), em parceria com o Instituto de Pesquisa Econômica Aplicada (IPEA) e a Fundação João Pinheiro (FJP) 12

Em virtude dos problemas de sub-registro e qualidade da informação de óbitos observados entre os municípios brasileiros 13, optou-se pela seleção de municípios segundo critérios de adequação da informação sobre óbitos. Esses critérios foram: população maior que 100 mil habitantes, população urbana maior que $90 \%$, desvio médio da taxa geral de mortalidade menor que $10 \%$, porcentagem de causas mal definidas menor que $10 \%$. Tais critérios foram aplicados para os três primeiros anos do estudo (1983-1985), a fim de minimizar possíveis vieses de seleção, já que nos anos mais recentes houve melhoria da qualidade da informação de saúde no país 13 . Dos 117 municípios selecionados como de boa qualidade da informação sobre óbitos, 108 estão localizados nas regiões Sul e Sudeste do país, caracterizadas como as de melhor nível sócioeconômico, de maior oferta e cobertura de serviços de saúde e menores níveis de mortalidade.

Considerando a importância da seleção de causas de morte, foram estudadas as classificações existentes na literatura de causas evitáveis por meio de ações como vacinação, tratamento preventivo, diagnóstico e tratamento médico apropriado. Foi construída uma proposta de seleção de causas baseada na classificação de Nolte \& McKee ${ }^{8}$, e nas propostas de Simonato et al. $14 \mathrm{e}$ de Tobias \& Jackson 15. A idade limite considerada para a maioria das causas foi 74 anos. Foram selecionadas causas de morte evitáveis distinguindose três grupos: aquelas evitáveis por diagnóstico e tratamento precoce, as evitáveis por melhoria no tratamento e na atenção médica, e a doença isquêmica do coração. Conforme pode ser visto na Tabela 1, a definição das causas de morte evitáveis restringiu-se aos diferentes componentes da atenção à saúde. Portanto, foram excluídas patologias cuja intervenção efetiva ocorre fora do controle direto do sistema de saúde, incluindo vários tipos de prevenção primária. Causas externas também não foram incluídas, embora se reconheça sua importância na sobremortalidade 
Tabela 1

Classificação de causas evitáveis de morte em grupos *.

\begin{tabular}{|c|c|c|c|}
\hline Grupo de causas & Idade (anos) & CID-9 ** & CID-10 *** \\
\hline \multicolumn{4}{|l|}{ Evitáveis por diagnóstico e tratamento precoce } \\
\hline Neoplasma maligno do colon e reto & $0-74$ & $153-154$ & $\mathrm{C} 18-\mathrm{C} 21$ \\
\hline Neoplasma maligno da pele & $0-74$ & 173 & C44 \\
\hline Neoplasma maligno da mama & $0-74$ & 174 & $\mathrm{C} 50$ \\
\hline Neoplasma maligno do colo do útero & $0-74$ & 180 & $\mathrm{C} 53$ \\
\hline Neoplasma maligno do corpo do útero & $0-44$ & 179,182 & $\mathrm{C} 54, \mathrm{C} 55$ \\
\hline Doenças da tireóide & $0-74$ & $240-246$ & E00-E07 \\
\hline Diabetes mellitus & $0-74$ & 250 & E10-E14 \\
\hline Epilepsia & $0-74$ & 345 & G40-G41 \\
\hline Doenças cerebrovasculares & $0-74$ & $430-438$ & $160-169$ \\
\hline Influenza & $0-74$ & 487 & J10-J11 \\
\hline Pneumonia & $0-74$ & $480-486$ & J12-J18 \\
\hline Hiperplasia da próstata & $0-74$ & 600 & N40 \\
\hline \multicolumn{4}{|l|}{ Evitáveis por melhoria no tratamento e na atenção médica } \\
\hline Infecções intestinais & $0-14$ & 001-009 & A00-A09 \\
\hline Tuberculose & $0-74$ & 010-018, 137 & A15-A19, B90 \\
\hline Outras infecções (difteria, tétano, poliomielite) & $0-74$ & $032,037,045$ & $\mathrm{~A} 36, \mathrm{~A} 35, \mathrm{~A} 80$ \\
\hline Coqueluche & $0-14$ & 033 & A37 \\
\hline Septicemia & $0-74$ & 38 & A40-A41 \\
\hline Sarampo & $1-14$ & 55 & B05 \\
\hline Neoplasma maligno do testículo & $0-74$ & 186 & C62 \\
\hline Doença de Hodgkin & $0-74$ & 201 & C81 \\
\hline Leucemia & $0-74$ & $204-208$ & C91-C95 \\
\hline Doença reumática crônica do coração & $0-74$ & $393-398$ & $105-109$ \\
\hline Doenças hipertensivas & $0-74$ & $401-405$ & $|10-| 13, \mid 15$ \\
\hline Todas as doenças respiratórias (excluindo pneumonia/influenza) & $1-14$ & $460-479,488-519$ & J00-J09, J20-J99 \\
\hline Úlcera péptica & $0-74$ & $531-533$ & K25-K27 \\
\hline Apendicite & $0-74$ & $540-543$ & K35-K38 \\
\hline Hérnia abdominal & $0-74$ & $550-553$ & $\mathrm{~K} 40-\mathrm{K} 46$ \\
\hline Colelitiase e colecistite & $0-74$ & $574-575.1$ & K80-K81 \\
\hline Nefrite e nefrose & $0-74$ & $580-589$ & $\begin{array}{c}\text { N00-N07, N17-N19, } \\
\text { N25-N27 }\end{array}$ \\
\hline Mortalidade materna & $0-74$ & $630-676$ & О00-О99 \\
\hline Anomalias congênitas do coração & $0-74$ & $745-747$ & Q20-Q28 \\
\hline $\begin{array}{l}\text { Acidentes com pacientes durante intervenção cirúrgica e atenção } \\
\text { médica }\end{array}$ & $0-74$ & E870-E876, E878-E879 & Y60-Y69, Y83-Y84 \\
\hline Doença isquêmica do coração & $0-74$ & $410-414$ & $120-\mid 25$ \\
\hline
\end{tabular}

* Seleção baseada em classificações propostas por Simonato et al. 14, Tobias \& Jackson 15 e Nolte \& McKee 8;

** Classificação Internacional de Doenças - 9ạ Revisão (1983-1995);

*** Classificação Internacional de Doenças - 10ạ Revisão (1996-2005).

masculina, especialmente da população jovem, e já tenha sido incorporada em tipologias mais recentes de causas evitáveis 14,15. Além disso, as causas perinatais não foram consideradas por falta de dados diretos disponíveis sobre nascidos vivos para os anos anteriores a 1994 .

A análise estatística foi realizada em três etapas. Na análise descritiva, foram calculadas as taxas de mortalidade padronizadas por idade, sendo utilizada, como distribuição etária padrão, a população do Brasil em 1991. Na segunda etapa, as análises de associação foram realizadas aplicando-se o modelo de regressão binomial negativa. A variável resposta foi o número de óbitos por causas evitáveis segundo sexo, e as variáveis explicativas selecionadas foram período (19831992 e 1993-2005), faixa etária, região brasileira e indicadores de condições sócio-econômicas. A 
primeira variável representa as mudanças experimentadas no sistema de saúde brasileiro, entendendo como marcador destas mudanças a introdução de normas em determinados momentos que redefiniram a organização de serviços de saúde no país 16 . Foram criados dois indicadores com base nas variáveis sócio-econômicas selecionadas, que explicavam $68 \%$ da variação observada nas variáveis originais. $\mathrm{O}$ primeiro fator, denominado "Desigualdade", apresentou correlação alta com as variáveis índice de Gini e razão entre a renda média dos $10 \%$ mais ricos e dos $40 \%$ mais pobres. O segundo fator refere-se ao "Nível Sócio-econômico", no qual foi encontrada uma alta correlação com as variáveis percentual de pessoas com idade igual ou maior do que 25 anos e 12 anos ou mais de estudo, percentual de pessoas com idade igual ou maior do que 25 anos e menos de 4 anos de estudo e renda per capita. Devido a problemas de qualidade da informação disponível, a questão da raça/etnia não foi introduzida no modelo de análise.

Assim, para a análise de associação, foi ajustado, para cada grupo de causas evitáveis, um modelo inicial com apenas uma variável e incluindo gradativamente cada uma das variáveis explicativas consideradas no estudo. Usando-se esse modelo, foi possível identificar as variáveis significativas nos diferentes grupamentos de causas evitáveis.

Na terceira etapa, foi aplicada a técnica de tábua de mortalidade de múltiplo decremento segundo o método dos riscos competitivos, formulado por Chiang 17, para avaliar a magnitude da incidência das causas evitáveis na probabilidade de morte, sobrevivência e esperança de vida para homens e mulheres. Essa técnica permite medir a influência de uma determinada causa de morte na evolução da esperança de vida ao nascer, assumindo como pressuposto que há vários riscos de morte agindo simultaneamente em cada indivíduo e que para cada risco existe uma força de mortalidade. As tábuas de vida de múltiplo decremento oferecem estimativas das probabilidades de morte com todas as causas (denominadas probabilidade bruta), e as probabilidades de morte se uma causa específica for retirada (denominadas probabilidade líquida). Assim, pressupõe-se que ao se remover uma causa de morte, a força das demais não seja afetada. Como a força de mortalidade total é o somatório das forças de mortalidade por várias causas, a diferença entre a estimativa da esperança de vida caso ninguém morresse por uma causa específica e a esperança de vida representa o tempo de vida médio da população sem a causa selecionada 17. Embora os pressupostos de independência e aditividade dos riscos possam suscitar questionamentos, estudos já demonstraram que o modelo proposto por Chiang é suficientemente plausível quando se pretende analisar o efeito da eliminação de uma causa de morte sobre os ganhos de esperança de vida de uma determinada população 18,19,20.

Na construção das tábuas de vida por sexo, utilizou-se o número médio de óbitos por idade para os anos de 2003-2005. Foram elaboradas tábuas de vida de múltiplo decremento por sexo para cada um dos três grupos de causas de morte evitáveis selecionados. Foram também calculadas as diferenças relativas entre as probabilidades de morte bruta e líquida por grupo de causas evitáveis segundo grupos etários para homens e mulheres. Uma vez que a idade limite da maioria das causas evitáveis selecionadas foi 74 anos, a análise baseia-se na expectativa de vida entre 0 nascimento e a idade de 74 anos, em vez da expectativa de vida ao nascer.

\section{Resultados}

No período de 1983 a 2005, as mortes evitáveis representaram cerca de $32 \%$ dos óbitos masculinos e $46 \%$ dos femininos. As causas evitáveis por diagnóstico e tratamento precoce responderam por $45 \%$ e $56 \%$ do total de óbitos por causas evitáveis para homens e mulheres, respectivamente (Tabela 2). Dentre essas, as doenças cerebrovasculares foram as mais freqüentes em ambos os sexos (em torno de $22 \%$ - dados não apresentados). As causas evitáveis por melhoria no tratamento e na atenção médica foram responsáveis por cerca de um quarto dos óbitos evitáveis em homens e mulheres. Nesse grupo de causas, as doenças hipertensivas foram as que tiveram a maior participação para ambos os sexos (em torno de 6\% - dados não apresentados). Já a doença isquêmica do coração foi responsável por cerca de $30 \%$ dos óbitos masculinos por causas evitáveis e $20 \%$ dos femininos.

Essas diferenças também se evidenciam ao se analisar os grupos etários: para as idades entre 0 e 14 anos, o percentual praticamente foi equivalente (29\% dos óbitos masculinos devem-se a causas evitáveis e $30 \%$ dos óbitos femininos). A maior diferença entre homens e mulheres quanto à participação das causas evitáveis no total de óbitos foi encontrada no grupo etário de 15 e 44 anos (38\% para as mulheres e $15 \%$ para os homens - dados não apresentados). Nas idades mais avançadas, a diferença não foi muito expressiva, sendo um pouco maior para as mulheres. Em relação às taxas específicas de mortalidade observa-se risco maior para os homens em todos os grupos de causas evitáveis, sendo que 
Distribuição dos óbitos por grupos de causas evitáveis segundo sexo. Brasil, 1983-2005.

\begin{tabular}{|c|c|c|c|c|}
\hline \multirow[t]{2}{*}{ Grupos de causas } & \multicolumn{2}{|c|}{ Homens } & \multicolumn{2}{|c|}{ Mulheres } \\
\hline & $\mathbf{n}$ & $\%$ & $\mathbf{n}$ & $\%$ \\
\hline Evitáveis por diagnóstico e tratamento precoce & 523.012 & 45,3 & 537.052 & 56,1 \\
\hline Evitáveis por melhoria no tratamento e na atenção médica & 285.928 & 24,8 & 231.501 & 24,2 \\
\hline Doença isquêmica do coração & 345.371 & 29,9 & 189.135 & 19,7 \\
\hline Total (causas evitáveis) & 1.154 .311 & 100,0 & 957.688 & 100,0 \\
\hline Total (todas as causas) & 3.591 .581 & & 2.089 .917 & \\
\hline \% evitáveis * & & 32,1 & & 45,8 \\
\hline
\end{tabular}

* Percentual em relação ao total (todas as causas).

esta diferença aumenta à medida que se avança na idade, particularmente após os 45 anos, e também para os óbitos por doença isquêmica do coração (dados não apresentados).

Ao analisar a evolução da mortalidade por causas evitáveis ao longo do período de 23 anos, observa-se que houve redução da participação das causas evitáveis no total de óbitos, maior para homens do que para mulheres (16\% e 9\%, respectivamente - dados não apresentados). Em relação aos riscos de morte, as taxas de mortalidade por causas evitáveis também indicam um descenso no período de 1983-2005, tanto para os homens quanto para as mulheres. A variação no período foi de $50 \%$ e $49 \%$, para homens e mulheres, respectivamente. Mas essa queda dos níveis de mortalidade refletiu pouco nos diferenciais entre os sexos, pois a razão de 1,3 entre as taxas de homens e mulheres manteve-se praticamente a mesma entre 1983 e 2005.

No modelo multivariado, os homens apresentaram risco maior de morrer em relação às mulheres em todos os três grupos de causas evitáveis, após controle das variáveis de confusão. Não houve alteração significativa nos resultados com o processo de inclusão seqüencial das variáveis de confusão no modelo. Entretanto, foram observadas variações entre os grupos de causas: ser do sexo masculino representou um risco $14 \%$ maior em relação ao sexo feminino para as causas evitáveis por diagnóstico e tratamento precoce, $30 \%$ maior para as causas evitáveis por melhoria no tratamento e na atenção médica e $117 \%$ para as mortes por doença isquêmica do coração (Tabela 3).

Usando-se a técnica de múltiplo decremento foram identificadas as mudanças no indicador esperança de vida com a eliminação total do efeito de cada um dos grupos de causas evitáveis (Tabela 4). Ao serem removidas as causas evi- táveis por diagnóstico e tratamento precoce, a esperança de vida entre o nascimento e 74 anos aumentou em quase 2 anos para os homens e em quase 5 para as mulheres. $\mathrm{O}$ segundo maior ganho na esperança de vida para os homens e mulheres se deu com a eliminação da doença isquêmica do coração (1,3 ano e 1,7 ano, respectivamente). Já a retirada das causas evitáveis por melhoria no tratamento e na atenção médica aumentou em quase 1 ano para os homens e em 1,5 ano para as mulheres.

Na Figura 1, são apresentados os diferenciais nas probabilidades de morte ao se eliminar um grupo de causas de morte em relação às probabilidades brutas. Para as mortes por causas evitáveis por diagnóstico e tratamento precoce, as maiores reduções para os homens ocorreram nas idades mais avançadas (acima de 40 anos). Já para as mulheres, o impacto passou a ser mais expressivo a partir dos 25 anos. Ressalta-se que as diferenças para esse grupo de causas evitáveis foram também importantes para as idades entre 1 e 4 anos em ambos os sexos. Para as causas evitáveis por melhoria no tratamento e na atenção médica, no caso da população masculina foram as crianças que sofreram um maior impacto nas probabilidades de morte, particularmente para as idades entre 1 e 9 anos. Para o sexo feminino, nesse mesmo grupo de causas, as diferenças entre as probabilidades de morte brutas e líquidas foram mais significativas nas crianças (especialmente entre 1 e 4 anos) e nas idades adultas jovens (até 34 anos). No caso das mortes por doença isquêmica do coração, o impacto só foi mais expressivo para as idades mais avançadas, a partir de 50 anos, sendo um pouco maior nos homens em relação às mulheres. 
Tabela 3

Razão de taxas de incidência de mortalidade masculina versus feminina por grupos de causas evitáveis ajustada por idade, período de análise, regiões e indicadores sociais. Brasil, 1983-2005.

\begin{tabular}{|c|c|c|c|c|c|c|}
\hline \multirow[t]{2}{*}{ Variáveis } & \multicolumn{2}{|c|}{$\begin{array}{l}\text { Óbitos por grupo de causas } \\
\text { evitáveis por diagnóstico } \\
\text { e tratamento precoce }\end{array}$} & \multicolumn{2}{|c|}{$\begin{array}{l}\text { Óbitos por grupo de causas } \\
\text { evitáveis por melhoria no } \\
\text { tratamento e na atenção médica }\end{array}$} & \multicolumn{2}{|c|}{$\begin{array}{c}\text { Óbitos por doença isquêmica } \\
\text { do coração }\end{array}$} \\
\hline & IRR * & IC95\% & IRR * & IC95\% & IRR * & IC95\% \\
\hline Ajustada por idade & 1,144 & $1,101-1,188$ & 1,301 & $1,249-1,356$ & 2,180 & $2,092-2,271$ \\
\hline Ajustada por idade e período de análise & 1,137 & $1,100-1,175$ & 1,300 & $1,253-1,349$ & 2,174 & $2,093-2,258$ \\
\hline $\begin{array}{l}\text { Ajustada por idade, período de análise } \\
\text { e regiões }\end{array}$ & 1,136 & $1,100-1,174$ & 1,300 & $1,258-1,343$ & 2,172 & $2,093-2,253$ \\
\hline $\begin{array}{l}\text { Ajustada por idade, período de análise, } \\
\text { regiões e nível sócio-econômico }\end{array}$ & 1,137 & $1,102-1,174$ & 1,303 & $1,263-1,344$ & 2,173 & $2,095-2,255$ \\
\hline $\begin{array}{l}\text { Ajustada por idade, período de análise, } \\
\text { regiões, nível sócio-econômico e } \\
\text { desigualdade }\end{array}$ & 1,137 & $1,102-1,174$ & 1,303 & $1,263-1,344$ & 2,174 & $2,095-2,255$ \\
\hline
\end{tabular}

IRR: incidence rate ratios (razão de taxas de incidência); resultados do modelo de regressão binomial negativa.

* Significativo a $0,01(p<0,01)$.

\section{Tabela 4}

Esperanças de vida entre nascimento e 74 anos observadas e estimadas eliminando o efeito dos grupos de causas de morte evitáveis segundo sexo. Brasil, 2003-2005.

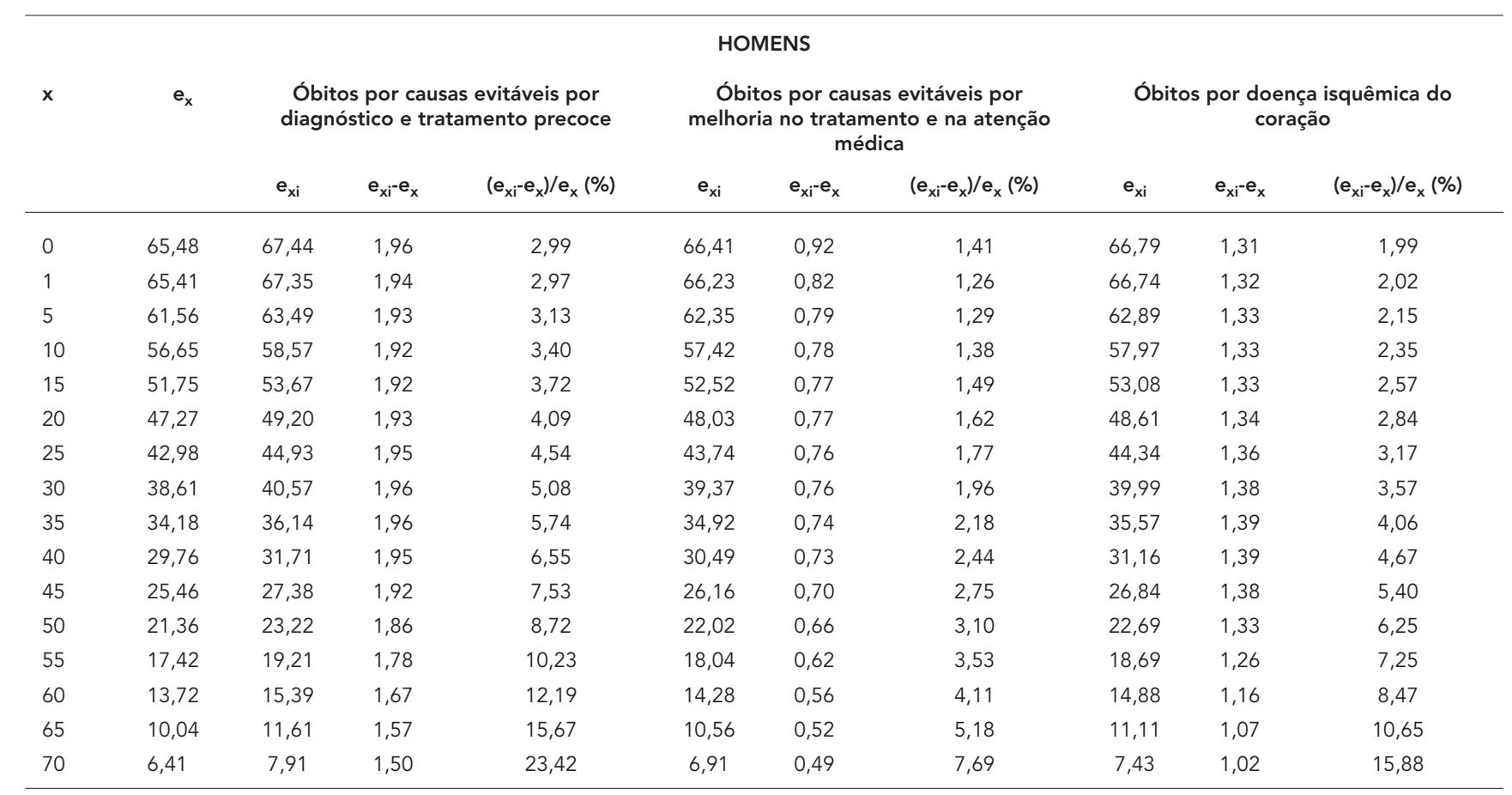

(continua) 


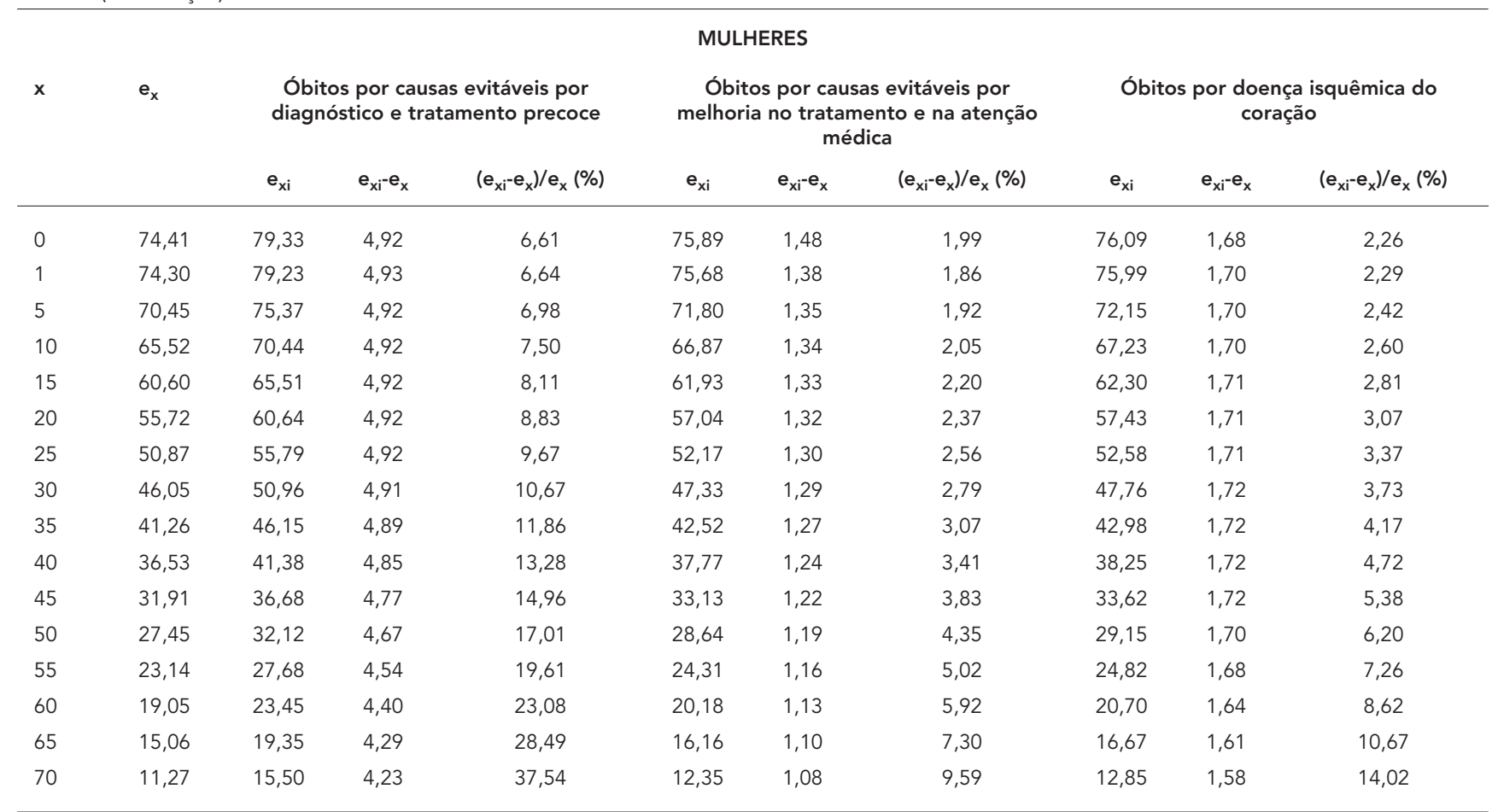

$e_{\mathrm{x}}$ : esperança de vida à idade $\mathrm{x}$ observada; $\mathrm{e}_{\mathrm{xi}}$ : esperança de vida à idade $\mathrm{x}$ estimada eliminando o efeito de grupo $\mathrm{x}$ de causas de morte evitáveis; $\mathrm{e}_{\mathrm{xi}}-\mathrm{e}_{\mathrm{x}}$ : diferença entre as esperanças de vida à idade $x$ observada e estimada eliminando o efeito do grupo x de causas de morte evitáveis; $\left(e_{x i} e_{x}\right) / e x$ : variação porcentual entre diferença das esperanças de vida à idade $x$ observada e estimada eliminando o efeito do grupo $x$ de causas de morte evitáveis.

\section{Discussão}

Os resultados apresentados neste trabalho indicam que houve uma queda nos níveis de mortalidade por causas evitáveis para homens e mulheres no período de 1983-2005. Entretanto, a diminuição observada afetou pouco os diferenciais entre os sexos. Ou seja, o risco de morte que era $34 \%$ maior para os homens do que para as mulheres em 1983, passou a ser $31 \%$ maior em 2005.

A sobremortalidade masculina ocorreu em todos os grupos de causas evitáveis após controle de variáveis demográficas, de nível sócioeconômico e a variável marcadora de mudanças na organização e oferta de assistência à saúde no período estudado. Chama atenção o expressivo risco de morte por doença isquêmica do coração entre os homens (117\% maior), após controle das variáveis de confusão. As taxas específicas foram de maior magnitude para as idades mais avançadas, e aqui também foram observados valores mais elevados para a população masculina.

Essa situação é refletida ainda no indicador esperança de vida entre o nascimento e 74 anos. As mulheres, que apresentavam uma sobrevida maior do que os homens, seriam mais benefi- ciadas caso se removessem as causas evitáveis, pois o ganho obtido na esperança de vida entre o nascimento e 74 anos seria maior para elas, o que levaria a um aumento no diferencial entre os sexos.

Assim, em que pese o fato de a participação relativa das causas evitáveis ser maior para as mulheres, todos os indicadores utilizados para analisar as diferenças entre homens e mulheres seguem uma mesma direção: na perspectiva da mortalidade por causas evitáveis, ser do sexo masculino representa um risco maior para qualquer um dos grupos de causas evitáveis selecionados no período de 1983 a 2005. O menor peso das causas evitáveis na distribuição proporcional de causas entre os homens provavelmente se deve à magnitude das mortes violentas, que contribuem em grande medida pelos diferenciais por sexo, resultado do padrão de urbanização e industrialização, característico particularmente das áreas mais desenvolvidas do país 3,21. Essa relevância das causas violentas entre os homens pode também ser uma explicação para o fato de que, ao aplicar o método de riscos competitivos, os ganhos na esperança de vida entre o nascimento e 74 anos foram sempre superiores para as mulheres do que para os homens 20 . 
Figura 1

Diferenças relativas entre as probabilidades de morte real e líquida por grupo de causas evitáveis, segundo faixas etárias. Brasil, 2003-2005.

1a) Homens

35

30

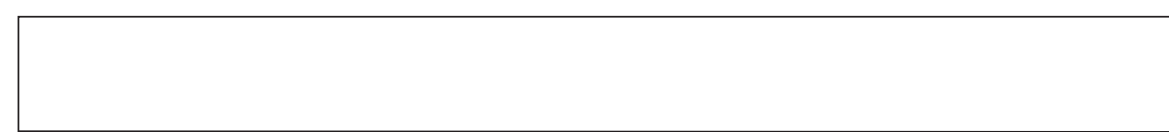

20

15

10

$\%$

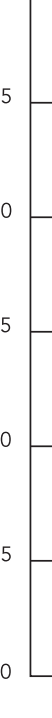

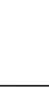
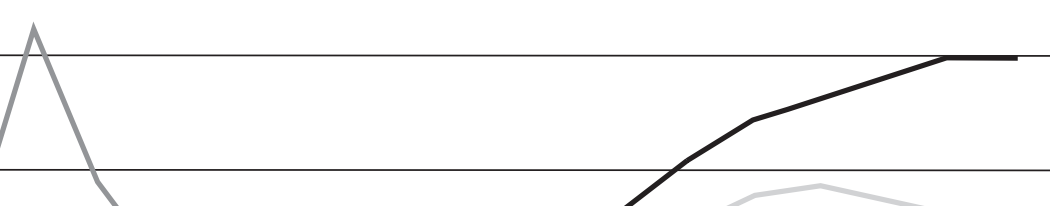

$\wedge$

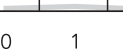

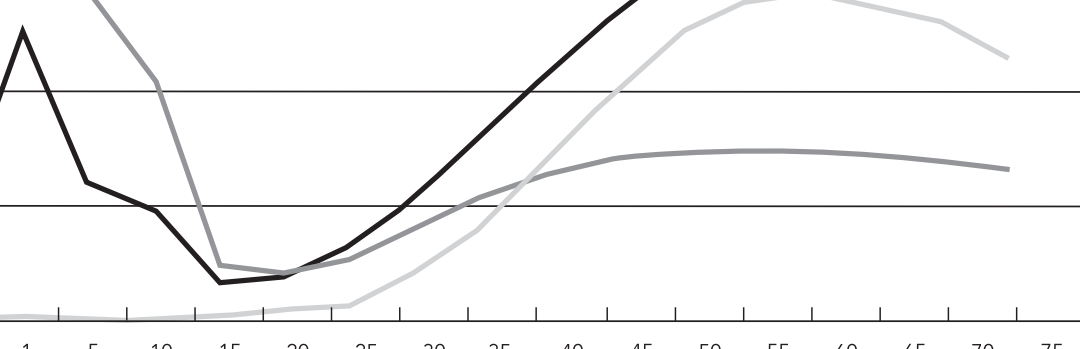
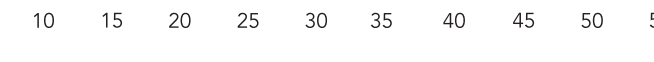

1b) Mulheres

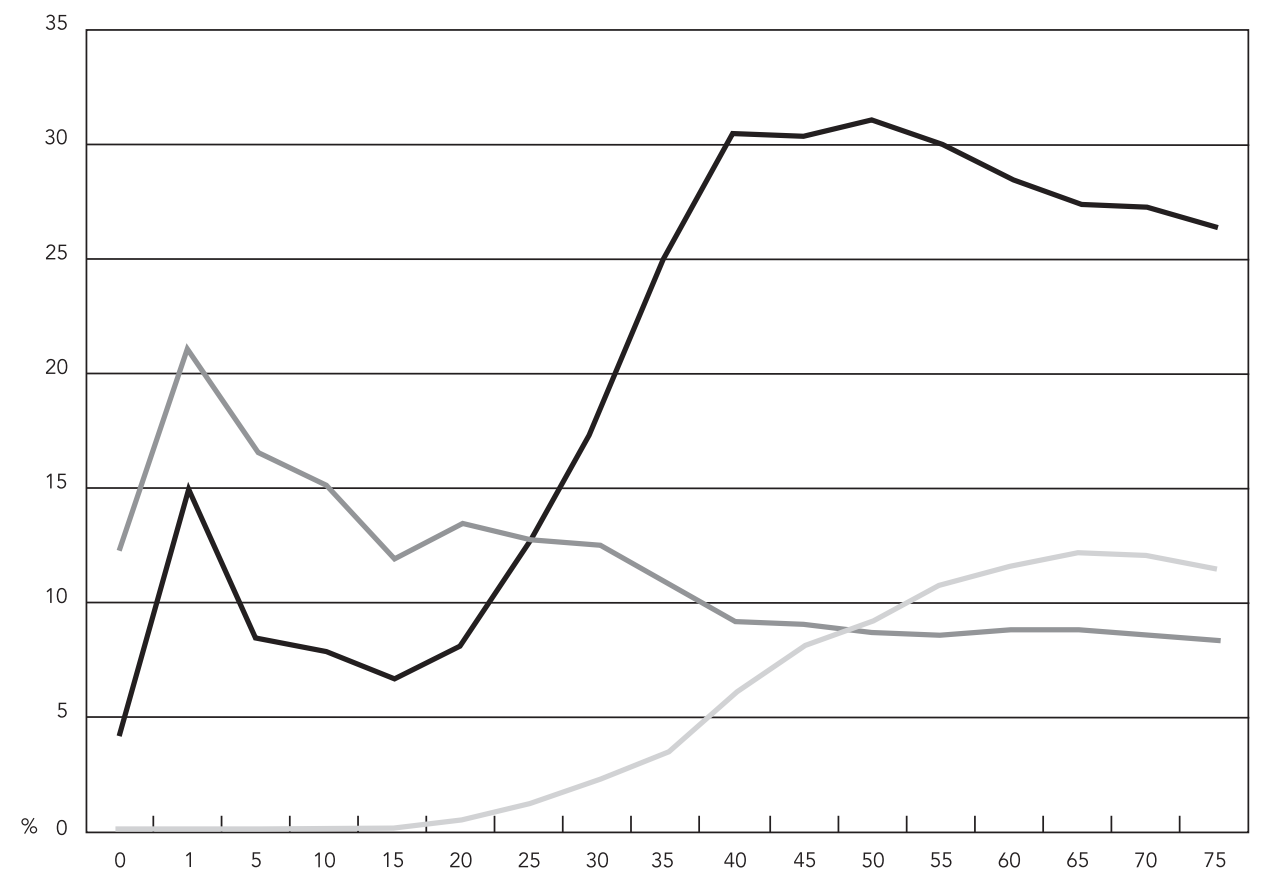

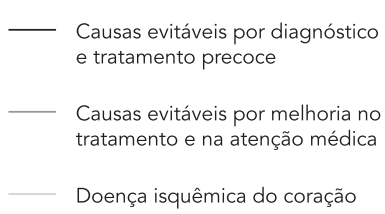

Grupos etários

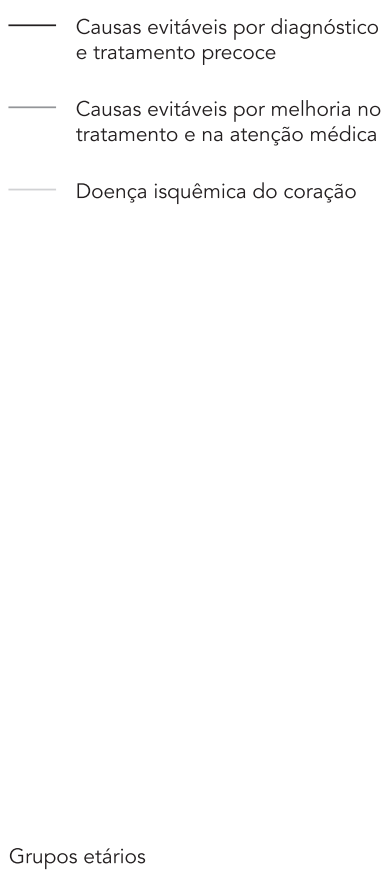


É importante considerar também que a composição dos grupos de causas evitáveis com peso de determinadas doenças diferenciado entre os sexos pode ter tido algum efeito sobre os resultados encontrados. De tal modo, a eliminação de causas evitáveis por diagnóstico e tratamento precoce teve uma maior influência para as mulheres do que para os homens. Esse é um grupo de causas no qual o peso entre as mulheres jovens é maior. Esse grupo reúne causas como doenças cerebrovasculares, neoplasias (mama, colo do útero, corpo de útero), pneumonia e diabetes mellitus, entre outras.

Já o grupo de causas evitáveis por melhoria no tratamento e atenção médica apresentou ganhos menores, mas com diferenciais importantes no risco de morte. Nesse grupo de causas estão, em virtude da sua evitabilidade, as infecções intestinais, cuja mortalidade é muito afetada por tratamento com antibióticos e vacinação, e outras reduzíveis por intervenções médicas e cirúrgicas, tais como as doenças hipertensivas, úlcera péptica, apendicite e as complicações da gravidez e parto. Isso pode explicar por que as diferenças entre as probabilidades de morte bruta e líquida são maiores paras as crianças e para a população feminina adulta jovem.

Cabe ressaltar que existem outros possíveis fatores que atuam sobre a mortalidade por causas evitáveis entre homens e mulheres. A adoção diferenciada de estilos de vida alternativos e mais saudáveis podem favorecer as mulheres 2,5, bem como a procura e utilização de serviços de saúde, sejam de promoção e prevenção como de assistência, que tende a ser maior entre a população feminina 22 . No caso da doença isquêmica do coração, é possível que o controle de fatores de risco entre homens e mulheres seja ainda mais diferenciado. Tradicionalmente, os homens apresentam maior risco por buscarem cuidados médicos mais tardiamente ou mesmo ignorarem os sintomas 23 .
Outro aspecto que não deve ser desconsiderado é que os diferenciais entre os sexos podem ocorrer de modo distinto nos vários grupos sociais. As parcelas mais carentes da população são as que estão mais expostas aos riscos de contrair e falecer por doenças susceptíveis de prevenir e curar, pois, em geral, estas doenças se relacionam a problemas em termos de níveis de vida, acesso aos serviços de saúde e ineficácia de programas de saúde pública ${ }^{24}$. Nesse caso, a variável sexo pode ser mais uma questão a ser analisada no enfoque da desigualdade social, pois os homens tendem a apresentar uma menor morbidade auto-referida do que as mulheres 25 , mas apresentam uma maior sobremortalidade.

Em síntese, os resultados deste estudo sugerem um declínio da mortalidade no Brasil por causas evitáveis por atenção à saúde entre 1983 e 2005, com importantes diferenciais segundo grupos de causas entre os sexos. Em razão das variações e diferenças encontradas, a análise dos diferenciais de mortalidade entre sexos por causas evitáveis pode se constituir numa ferramenta oportuna para monitorar a situação de saúde de populações masculinas e femininas. Além disso, deve-se considerar a importância de avançar na análise de fatores relacionados à questão de gênero que podem estar associados ao risco diferenciado de morte por causas evitáveis entre homens e mulheres 26. Há ainda aspectos regionais, sócio-econômicos e culturais que devem ser incorporados aos estudos de mortalidade evitável. É importante considerar não somente as questões de acesso aos serviços de saúde, em alguma medida já equacionadas nos municípios maiores, mas de qualidade da assistência prestada, utilizando-se outros indicadores que possam captar com maior propriedade o impacto de ações e programas específicos sobre as causas de morte evitáveis. Reduzir as desigualdades em saúde vai depender em parte de políticas que privilegiem e garantam intervenções que incorporem novas abordagens de prevenção e promoção à saúde. 


\section{Resumo}

O objetivo do estudo foi analisar a mortalidade de homens e mulheres em municípios brasileiros entre 1983 e 2005, segundo três grupamentos de causas de morte evitáveis: evitáveis por diagnóstico e tratamento precoce, evitáveis por melhoria no tratamento e na atenção médica e doença isquêmica do coração. A associação entre a mortalidade evitável e as variáveis selecionadas foi realizada usando-se o modelo de regressão binomial negativa. Avaliou-se a magnitude da incidência das causas evitáveis na esperança de vida por meio da técnica de tábua de mortalidade de múltiplo decremento. Os homens apresentaram um risco maior de morrer em relação às mulheres para os grupos de causas evitáveis estudados, após controle de variáveis selecionadas. O ganho na esperança de vida ao nascer é sempre maior para as mulheres, com um aumento de até 5 anos para elas, ao eliminar as causas evitáveis por diagnóstico e tratamento precoce. Deve-se avançar na análise de fatores relacionados à questão de gênero, que podem estar associados ao risco diferenciado de morte entre os sexos.

Mortalidade; Causas de Morte; Homens; Mulheres

\section{Colaboradores}

D. M. X. Abreu participou do delineamento do estudo realizou o tratamento e análise dos dados, discussão dos resultados e redação final do artigo. E. B. França orientou o delineamento do estudo, participou da análise e discussão dos resultados e da redação final do trabalho. C. C. César colaborou na análise dos dados e revisão final do artigo.

\section{Agradecimentos}

D. M. X. Abreu agradece a bolsa de estudos recebida do Programa de Doutorado com Estágio no Exterior da Coordenação de Aperfeiçoamento de Pessoal de Nível Superior (CAPES; processo CAPES BEX2632/06-3).

\section{Referências}

1. Trovato F, Heyen NB. A varied pattern of change of the sex differential in survival in the G7 countries. J Biosoc Sci 2006; 38:391-401.

2. Wong MD, Chung AK, Boscardin WJ, Li M, Hsieh HJ, Ettner SL, et al. The contribution of specific causes of death to sex differences in mortality. Public Health Rep 2006; 121:746-54

3. Chor D, Duchiade MP, Jourdan AMF. Diferencial de mortalidade em homens e mulheres em localidade da região Sudeste, Brasil: 1960, 1970 e 1980. Rev Saúde Pública 1992; 26:246-55.

4. Case A, Paxson C. Sex differences in morbidity and mortality. Demography 2005; 42:189-214.

5. Fiala J, Brázdová Z. A comparison between the lifestyles of men and women-parents of school age children. Cent Eur J Public Health 2000; 8:94-100.

6. Oliveira JC, Albuquerque FRPC, Senna JRX. Breves notas sobre a mortalidade no Brasil no período 2000-2005. http://www.ibge.gov.br/home/estatis tica/populacao/tabuadevida/2005/notatecnica. pdf (acessado em 17/Ago/2007).

7. Abreu DMX, Rodrigues RN. Diferenciais de mortalidade entre as regiões metropolitanas de Belo Horizonte e Salvador, 1985-1995. Rev Saúde Pública 2000; 34:514-21.

8. Nolte E, McKee M. Does health care save lives? Avoidable mortality revisited. London: Nuffield Trust; 2004. 
9. Lopez A. The sex mortality differential in developed countries. In: Lopez A, Ruzicka L, editors. Sex differentials in mortality: trends, determinants and consequences Canberra: Australian National University Press; 1983. (Department of Demography, Miscellaneous Series, 4).

10. Cordeiro H. Descentralização, universalidade e eqüidade nas reformas da saúde. Ciênc Saúde Coletiva 2001; 6:319-28.

11. Abreu DMX, César CC, França EB. Relação entre as causas de morte evitáveis por atenção à saúde e a implementação do Sistema Único de Saúde no Brasil. Rev Panam Salud Pública 2007; 21:282-91.

12. Programa das Nações Unidas para o Desenvolvimento/Instituto de Pesquisa Econômica Aplicada/ Fundação João Pinheiro. Atlas do desenvolvimento humano no Brasil. http://www.pnud.org.br/atlas/ (acessado em 20/Jul/2005).

13. Departamento de Análise de Situação de Saúde, Secretaria de Vigilância em Saúde, Ministério da Saúde. Saúde Brasil 2004 - uma análise da situação de saúde. Brasília: Ministério da Saúde; 2004.

14. Simonato L, Ballard T, Bellini P, Winkelmann R. Avoidable mortality in Europe 1955-1994: a plea for prevention. J Epidemiol Community Health 1998; 52:624-30.

15. Tobias M, Jackson G. Avoidable mortality in New Zealand, 1981-97. Aust N Z J Public Health 2001; 25:12-20.

16. Levcovitz E, Lima LD, Machado CV. Política de saúde nos anos 90: relações intergovernamentais e papel das Normas Operacionais Básicas. Ciênc Saúde Coletiva 2001; 6:269-91.

17. Chiang CL. Competing risks in mortality analysis. Ann Rev Public Health 1991; 12:281-307.
18. Barboni AR, Gotlieb SLD. Impacto de causas básicas de morte na esperança de vida em Salvador e São Paulo, 1996. Rev Saúde Pública 2004; 38:16-23.

19. Silva MGC. Ganhos potenciais em esperança de vida com a exclusão das mortes por neoplasias malignas em Fortaleza, 1993-95. Rev Bras Cancerol 2001; 47:413-24.

20. Santos JLF, Ortiz LP, Yazaki LM. Aplicações da técnica de riscos competitivos a dados brasileiros. In: Anais do IV Encontro Nacional de Estudos Populacionais da ABEP. http://www.abep.nepo.unicamp. br/docs/anais/pdf/1984/T84V02A21.pdf (acessado em 05/Ago/2007).

21. Aquino EML, Menezes GM, Amoedo MBE, Nobre LCC. Mortalidade feminina no Brasil: sexo frágil ou sexo forte? Cad Saúde Pública 1991; 7:174-89.

22. Westerling R. Decreasing gender differences in "avoidable" mortality in Sweden. Scand J Public Health 2003; 31:342-9.

23. Daniel E, Germiniani H, Nazareno ER, Braga SV, Winkler AM, Cunha CL. Tendência da mortalidade por doenças isquêmicas do coração na cidade de Curitiba - Brasil, de 1980 a 1998. Arq Bras Cardiol 2005; 85:100-4.

24. Silva JB, Barros MBA. Epidemiologia e desigualdade: notas sobre a teoria e a história. Rev Panam Salud Pública 2002; 12:375-83.

25. Mendoza-Sassi RA, Béria JU. Gender differences in self-reported morbidity: evidence from a population-based study in southern Brazil. Cad Saúde Pública 2007; 23:341-6.

26. Jougla E, Pavillon G, Lefèvre H, Toullec A. Gender differences in premature mortality and avoidable deaths. Rev Epidemiol Sante Publique 2004; 52:317-28.

Recebido em 08/Set/2008

Versão final reapresentada em 16/Jun/2009

Aprovado em 11/Set/2009 